\title{
First-order valence transition: neutron diffraction, inelastic neutron scattering, and $x$-ray absorption investigations on the double perovskite $\mathrm{Ba}_{2} \mathrm{PrRu}_{0.9} \mathrm{Ir}_{0.1} \mathrm{O}_{6}$
}

\author{
J. Sannigrahi, D. T. Adroja, C. Ritter, W. Kockelmann, \\ A. D. Hillier, K. S. Knight, A. T. Boothroyd, \\ M. Wakeshima, Y. Hinatsu, J. F. W. Mosselmans, \\ and S. Ramos
}

\section{Published version information}

Citation: J Sannigrahi et al. "First-order valence transition: neutron diffraction, inelastic neutron scattering, and $x$-ray absorption investigations on the double perovskite $\mathrm{Ba}_{2} \mathrm{PrRu}_{0.9} \mathrm{I}_{0.1} \mathrm{O}_{6}$." Physical Review B, vol. 99, no. 18 (2019): 184440.

DOI: $\underline{10.1103 / P h y s R e v B .99 .184440}$

This version is made available in accordance with publisher policies. Please cite only the published version using the reference above. This is the citation assigned by the publisher at the time of issuing the APV. Please check the publisher's website for any updates. 


\title{
First-order valence transition: Neutron diffraction, inelastic neutron scattering, and $x$-ray absorption investigations on the double perovskite $\mathrm{Ba}_{2} \operatorname{PrRu}_{0.9} \operatorname{Ir}_{0.1} \mathrm{O}_{6}$
}

\author{
J. Sannigrahi,,${ }^{1,}$ D. T. Adroja,${ }^{1,2, \dagger}$ C. Ritter, ${ }^{3}$ W. Kockelmann, ${ }^{1}$ A. D. Hillier, ${ }^{1}$ K. S. Knight, ${ }^{1}$ A. T. Boothroyd, ${ }^{4}$ \\ M. Wakeshima, ${ }^{5}$ Y. Hinatsu, ${ }^{5}$ J. F. W. Mosselmans, ${ }^{6}$ and S. Ramos 6,7 \\ ${ }^{1}$ ISIS Facility, Rutherford Appleton Laboratory, STFC, Chilton, Didcot, OX11 0QX, United Kingdom \\ ${ }^{2}$ Highly Correlated Matter Research Group, Physics Department, University of Johannesburg, P.O. Box 524, \\ Auckland Park 2006, South Africa \\ ${ }^{3}$ Institut Laue Langevin, 71 Rue des Martyrs, 38042 Grenoble, France \\ ${ }^{4}$ Department of Physics, University of Oxford, Clarendon Laboratory, Parks Road, Oxford, OX1 3PU, United Kingdom \\ ${ }^{5}$ Hokkaido University School of Science, Chemistry Department, Sapporo, Hokkaido 0600810, Japan \\ ${ }^{6}$ Diamond Light Source, Harwell Science \& Innovation Campus, Didcot, Oxon OX11 ODE, United Kingdom \\ ${ }^{7}$ School of Physical Sciences, University of Kent, Canterbury, Kent, CT2 7NH, United Kingdom
}

(Received 15 March 2019; revised manuscript received 26 April 2019; published 28 May 2019)

\begin{abstract}
Bulk studies have revealed a first-order valence phase transition in $\mathrm{Ba}_{2} \operatorname{PrRu}_{1-x} \operatorname{Ir}_{x} \mathrm{O}_{6}(0.10 \leqslant x \leqslant 0.25)$, which is absent in the parent compounds with $x=0\left(\operatorname{Pr}^{3+}\right)$ and $x=1\left(\operatorname{Pr}^{4+}\right)$, which exhibit antiferromagnetic order with transition temperatures $T_{\mathrm{N}}=120$ and $72 \mathrm{~K}$, respectively. In the present study, we have used magnetization, heat capacity, neutron diffraction, inelastic neutron scattering, and $\mathrm{x}$-ray absorption measurements to investigate the nature of the Pr ion in $x=0.1$. The magnetic susceptibility and heat capacity of $x=0.1$ show a clear sign of the first-order valence phase transition below $175 \mathrm{~K}$, where the Pr valence changes from $3+$ to $4+$. Neutron diffraction analysis reveals that $x=0.1$ crystallizes in a monoclinic structure with space group $P 2_{1} / n$ at $300 \mathrm{~K}$, but below $175 \mathrm{~K}$ two phases coexist, the monoclinic having the $\mathrm{Pr}$ ion in a $3+$ valence state and a cubic one $(F m \overline{3} m)$ having the Pr ion in a $4+$ valence state. Clear evidence of an antiferromagnetic ordering of the Pr and $\mathrm{Ru}$ moments is found in the monoclinic phase of the $x=0.1$ compound below $110 \mathrm{~K}$ in the neutron diffraction measurements. Meanwhile, the cubic phase remains paramagnetic down to $2 \mathrm{~K}$, a temperature below which heat capacity and susceptibility measurements reveal a ferromagnetic ordering. High energy inelastic neutron scattering data reveal well-defined high-energy magnetic excitations near $264 \mathrm{meV}$ at temperatures below the valence transition. Low energy INS data show a broad magnetic excitation centered at $50 \mathrm{meV}$ above the valence transition, but four well-defined magnetic excitations at $7 \mathrm{~K}$. The high energy excitations are assigned to the $\operatorname{Pr}^{4+}$ ions in the cubic phase and the low energy excitations to the $\mathrm{Pr}^{3+}$ ions in the monoclinic phase. Further direct evidence of the Pr valence transition has been obtained from the x-ray absorption spectroscopy. The results on the $x=0.1$ compound are compared with those for $x=0$ and 1 .
\end{abstract}

DOI: 10.1103/PhysRevB.99.184440

\section{INTRODUCTION}

In metallic solids there is a general correlation between the loss of magnetism and the decrease or increase of the unitcell volume. This tendency is especially pronounced in $\mathrm{Ce}$ and $\mathrm{Yb}$ compounds, which have nearly degenerate electronic configurations, $4 f^{n}$ and $4 f^{n+1}$, where $n$ is the number of electrons in the $4 f$ shell [1-6]. Due to the presence of two nearly degenerate states, these compounds display intermediate valence or nonintegral valence behavior. Generally the intermediate valence systems exhibit a gradual change in the valence state of the rare-earth ions with temperature, pressure, and alloying [7-10]. However, there exist a handful of systems [11-13] in which the valence transition is of first order as a function of temperature or pressure while crystal symmetry remains the same below and above the phase transition. A

\footnotetext{
*jhuma.sannigrahi@stfc.ac.uk

$\dagger$ devashibhai.adroja@stfc.ac.uk
}

classic example is Ce metal, which shows a pressure induced first-order isostructural transition (at $8 \mathrm{kbar}$ at $300 \mathrm{~K}$ ) from a magnetic $\gamma$ phase to a nonmagnetic $\beta$ phase [14].

Very recently, it has been shown through magnetic susceptibility measurements that $\mathrm{Ba}_{2} \mathrm{PrRu}_{1-x} \mathrm{Ir}_{x} \mathrm{O}_{6}$ (with $0.1 \leqslant$ $x \leqslant 0.25$ ) compounds [15] also exhibit a temperature-induced valence transition, similar to those observed in $\mathrm{CeNi}_{1-x} \mathrm{Co}_{x} \mathrm{Sn}$ (Ref. [16]) and $\mathrm{YbInCu}_{4}$ (Ref. [17]). Unlike in the Ce and $\mathrm{Yb}$ compounds, however, in which one valence state $\left(\mathrm{Ce}^{3+} / \mathrm{Yb}^{3+}\right)$ is magnetic and the other $\left(\mathrm{Ce}^{4+} / \mathrm{Yb}^{2+}\right)$ has zero magnetic moment $[11,13]$, the valence phase transition in the Pr compounds is especially interesting as both $\operatorname{Pr}^{3+}$ and $\operatorname{Pr}^{4+}$ carry a magnetic moment.

$\mathrm{Ba}_{2} \mathrm{PrRu}_{1-x} \mathrm{Ir}_{x} \mathrm{O}_{6}$ compounds crystallize in the perovskitetype structure, with a small monoclinic distortion, and have a $1: 1$ ordered arrangement between $\mathrm{Pr}^{3+}$ and $\mathrm{Ru}^{5+}$ (or $\mathrm{Ir}^{5+}$ ) over the six-coordinate $B$ sites of the well known perovskite $\mathrm{ABO}_{3}$ structure $[15,18]$. The lattice parameters and unit cell volume of the $\mathrm{Ru}$-rich side of $\mathrm{Ba}_{2} \mathrm{PrRu}_{1-x} \mathrm{Ir}_{x} \mathrm{O}_{6}$ decrease with increasing Ir concentration, while for the Ir-rich side they are 
nearly constant [18]. The change in the lattice parameters and unit cell volume is attributed to the change in the oxidation state of the Prion.

A previous neutron diffraction study on $\mathrm{Ba}_{2} \mathrm{PrRuO}_{6}$ revealed type-I antiferromagnetic ordering of $\mathrm{Pr}^{3+}$ and $\mathrm{Ru}^{5+}$ moments below $T_{\mathrm{N}} \simeq 120 \mathrm{~K}[19,20]$. Zero-field muon-spin rotation $(\mu \mathrm{SR})$ measurements on $\mathrm{Ba}_{2} \operatorname{PrRu}_{1-x} \mathrm{Ir}_{x} \mathrm{O}_{6}$ with $x=$ $0,0.1$, and 1.0 revealed in addition a coexistence of two phases in $x=0.1$ below the valence phase transition $\left(T_{\mathrm{V}} \simeq\right.$ $170 \mathrm{~K}$ ), one phase being magnetically ordered and the other paramagnetic [21].

The aim of the present study was to obtain direct information on the valence phase transition and changes in the crystal structure, as well as details of the magnetic structure below the ordering temperature. To this end, high resolution and high intensity neutron powder diffraction, as well as inelastic neutron scattering measurements, were performed on $\mathrm{Ba}_{2} \mathrm{PrRu}_{0.9} \mathrm{Ir}_{0.1} \mathrm{O}_{6}$. Here we shall report how the valence state transition transforms the ground state magnetic structure in this compound as well as the nature of the crystal field ground state of the $\mathrm{Pr}^{4+}$ ion. For comparison we also present additional results on $\mathrm{Ba}_{2} \mathrm{PrRuO}_{6}$.

\section{EXPERIMENTAL DETAILS}

Polycrystalline samples of $\mathrm{Ba}_{2} \mathrm{PrRu}_{0.9} \mathrm{Ir}_{0.1} \mathrm{O}_{6}$ were prepared following a solid state reaction route in air. A stoichiometric mixture of $\mathrm{BaCO}_{3}, \mathrm{RuO}_{2}$, Ir metal, and $\operatorname{Pr}_{6} \mathrm{O}_{11}$ was initially mixed homogeneously in the stoichiometric ratio, pelletized, and calcined at $900^{\circ} \mathrm{C}$ for $48 \mathrm{~h}$. The final sintering was performed at $1200^{\circ} \mathrm{C}$ for 3 days with several intermediate processes of regrinding and repelletizing [15].

The magnetic susceptibility $(\chi)$ and heat capacity $\left(C_{p}\right)$ measurements were made on a Quantum Design physical properties measurement system (PPMS). Neutron powder diffraction patterns for $\mathrm{Ba}_{2} \operatorname{PrRu}_{0.9} \mathrm{Ir}_{0.1} \mathrm{O}_{6}$ were recorded on the time-of-flight (TOF) High Resolution Powder Diffractometer (HRPD) at the ISIS Neutron and Muon Facility, UK. Data were collected in the temperature range 4 to $300 \mathrm{~K}$. The sample was finely ground and was contained in a thin walled vanadium can. The analysis of HRPD data was carried out with the GSAS software [22] and guided by group-subgroup relationships of ordered double perovskites. The background was defined by a third order Chebychev polynomial in TOF in the Rietveld refinements. Further neutron diffraction measurements to investigate the magnetic structure were performed on the high intensity diffractometer D20 at the Institut LaueLangevin, Grenoble, France, with a constant wavelength of $2.41 \AA$.

Inelastic neutron scattering (INS) measurements were carried out on the High Energy Transfer (HET) TOF spectrometer at the ISIS Facility with incident neutron energies $\left(E_{\mathrm{i}}\right)$ of $900,500,200,100,60$, and $15 \mathrm{meV}$ at temperatures of $222 \mathrm{~K}$ (above $T_{\mathrm{N}}$ ) and $5 \mathrm{~K}$ (below $T_{\mathrm{N}}$ ). The powder sample with $x=0.1$ was mounted in a thin Al-foil envelope, which was cooled down to $5 \mathrm{~K}$ in a closed cycle refrigerator (CCR) in the presence of helium exchange gas.

The $\operatorname{Pr} L_{3}$-edge $\mathrm{x}$-ray absorption near-edge structure (XANES) of $x=0,0.1$, and 1 compounds was measured in transmission mode between $77 \mathrm{~K}$ and $300 \mathrm{~K}$ on the beamline
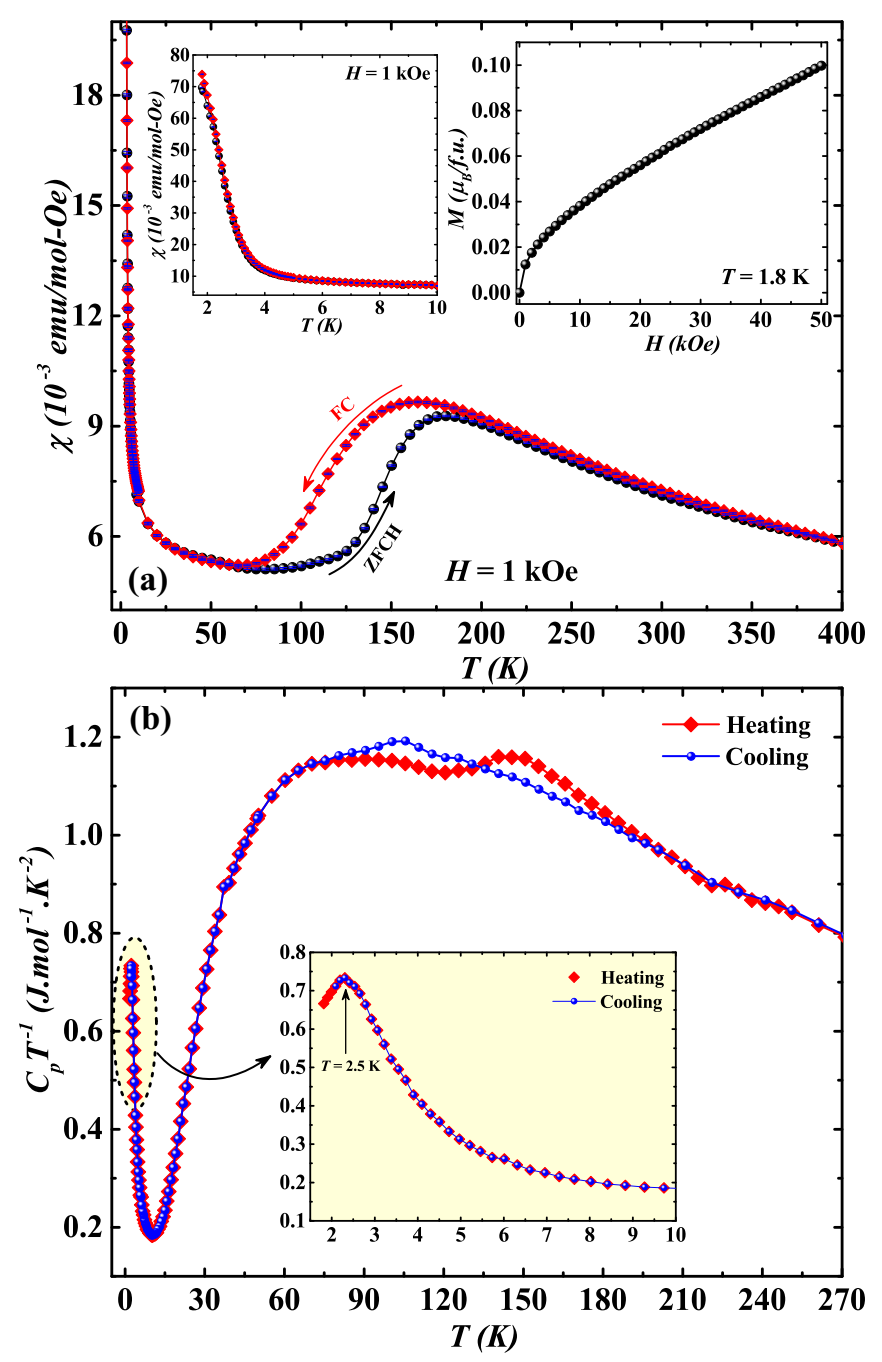

FIG. 1. (a) Temperature variation of the ZFC and FC susceptibility of $\mathrm{Ba}_{2} \operatorname{PrRu}_{0.9} \mathrm{Ir}_{0.1} \mathrm{O}_{6}$ in a measuring field of $H=1 \mathrm{kOe}$. The top left inset shows the susceptibility behavior at low temperature, indicating ferromagnetic ordering below $3 \mathrm{~K}$, and the top right inset shows the magnetization isotherm at $1.8 \mathrm{~K}$. (b) Heat capacity data, plotted as $C_{p} / T$ vs $T$, taken during heating and cooling cycles. The inset shows the low temperature behavior of $C_{p} / T$ vs $T$, which indicates a magnetic anomaly near $2.5 \mathrm{~K}$.

B18, the Core EXAFS (Extended X-ray Absorption Fine Structure) Beamline, at the Diamond Light Source, UK, and at station 7.1 of the Synchrotron Radiation Source (SRS) at the Daresbury Laboratory, UK. Samples were prepared by grinding the polycrystalline material into a fine powder, mixing it with cellulose, and pressing the mixture into pellets.

\section{RESULTS AND DISCUSSION}

\section{A. Magnetization and heat capacity}

Figure 1(a) shows the temperature dependence of magnetic susceptibility of $\mathrm{Ba}_{2} \mathrm{PrRu}_{0.9} \mathrm{Ir}_{0.1} \mathrm{O}_{6}$ in an applied magnetic field of $H=1 \mathrm{kOe}$. This compound shows a distinct magnetic anomaly between 60 and $200 \mathrm{~K}$. A drop in susceptibility is observed on cooling below $200 \mathrm{~K}$ for both zero-fieldcooled (ZFC) and field-cooled (FC) cycles. Clear hysteresis 
between ZFC and FC data signifies the first-order nature of this transition. This anomaly is ascribed to the valence phase transition of the Pr ions on the B site. [15] The temperature range in which the ZFC and FC data separate from each other is the same as the temperature range in which two perovskite phases $\mathrm{Ba}_{2} \mathrm{Pr}^{3+}(\mathrm{Ru}, \mathrm{Ir})^{5+} \mathrm{O}_{6}$ and $\mathrm{Ba}_{2} \mathrm{Pr}^{4+}(\mathrm{Ru}, \mathrm{Ir})^{4+} \mathrm{O}_{6}$ coexist in accordance with $\mathrm{X}$-ray diffraction measurements [18]. This shows that the valence state of the Pr ion changes from $\mathrm{Pr}^{3+}$ (above $200 \mathrm{~K}$ ) to $\mathrm{Pr}^{4+}$ below $200 \mathrm{~K}$. The inset (top right) of Fig. 1(a) shows the isothermal magnetization recorded at $1.8 \mathrm{~K}$ after ZFC. At low fields the magnetization shows ferromagnetic behavior without hysteresis. The value of the saturated moment at $50 \mathrm{kOe}$ is approximately $0.1 \mu_{\mathrm{B}} / \mathrm{f}$.u.

The magnetic anomaly between 60 and $200 \mathrm{~K}$ is also clearly present in the temperature dependent heat capacity data shown in the main panel of Fig. 1(b). The cooling and heating curves start to separate below $200 \mathrm{~K}$, consistent with the first-order nature of the transition. Interestingly, we observed a maximum in the $C_{p} / T$ versus $T$ curve at $\approx 2.5 \mathrm{~K}$ [inset of Fig. 1(b)] which coincides with the sharp rise in the magnetic susceptibility below $3 \mathrm{~K}$ [inset of Fig. 1(a)] indicating ferromagnetic ordering. The origin of the ferromagnetic ordering is attributed to the ordering of the cubic phase in the two-phase separated states, as will be discussed in Sec. B 2.

\section{B. Neutron diffraction}

\section{Crystal structure}

The temperature dependence of the powder neutron diffraction patterns of $\mathrm{Ba}_{2} \operatorname{PrRu}_{0.9} \mathrm{Ir}_{0.1} \mathrm{O}_{6}$ as obtained from HRPD is shown in Fig. 2(a), with heating cycles between 5 and $300 \mathrm{~K}$. At $300 \mathrm{~K}$, Fig. 2(c), the data can be refined using a single monoclinic phase with space group $P 2_{1} / n$. As the temperature is lowered, extra peaks arise below about $170 \mathrm{~K}$ and persist down to $5 \mathrm{~K}$, as shown in Fig. 2(a). Rietveld analysis shows that these extra peaks are due to the appearance of a new phase, which was identified as a cubic structure with space group $F m \overline{3} m$. A two-phase fit at $5 \mathrm{~K}$ is shown in Fig. 2(b). This new phase is therefore similar to the parent phase $\mathrm{Ba}_{2} \mathrm{PrIrO}_{6}$ (with $\mathrm{Pr}^{4+}$ ), implying that the $\operatorname{Pr}$ ion in the cubic phase of $\mathrm{Ba}_{2} \operatorname{PrRu}_{0.9} \mathrm{Ir}_{0.1} \mathrm{O}_{6}$ is in the $\operatorname{Pr}^{4+}$ state below $T_{\mathrm{V}} \simeq 170 \mathrm{~K}$, the valence phase transition temperature $[23,24]$. It is to be noted that the $\mathrm{Pr}$ ions are in the $\mathrm{Pr}^{3+}$ state in $\mathrm{Ba}_{2} \mathrm{PrRuO}_{6}$ and also in $\mathrm{Ba}_{2} \mathrm{PrRu}_{0.9} \mathrm{Ir}_{0.1} \mathrm{O}_{6}$ at $300 \mathrm{~K}$. As the cubic phase Bragg reflections appear on cooling below $T_{\mathrm{V}}$ there is a concomitant decrease in the reflections corresponding to the room temperature monoclinic phase; see Fig. 2(a).

In order to describe the crystal structures more quantitatively we performed detailed structural analysis via Rietveld refinement of the high resolution powder neutron diffractograms recorded between 5 and $300 \mathrm{~K}$. Figure 3(a) shows how the fraction of cubic and monoclinic phases changes with temperature. At $5 \mathrm{~K}$ the estimated volume faction is $58 \%$ for the cubic phase and $42 \%$ for the monoclinic phase. Above $125 \mathrm{~K}$ the fraction of the monoclinic phase sharply increases and the cubic phase becomes almost imperceptible above $170 \mathrm{~K}$. The temperature dependence of the monoclinic lattice parameters and $\beta$ angle are shown in Figs. 3(b), 3(c) and 3(e). It can be seen that with decreasing cubic phase fraction the
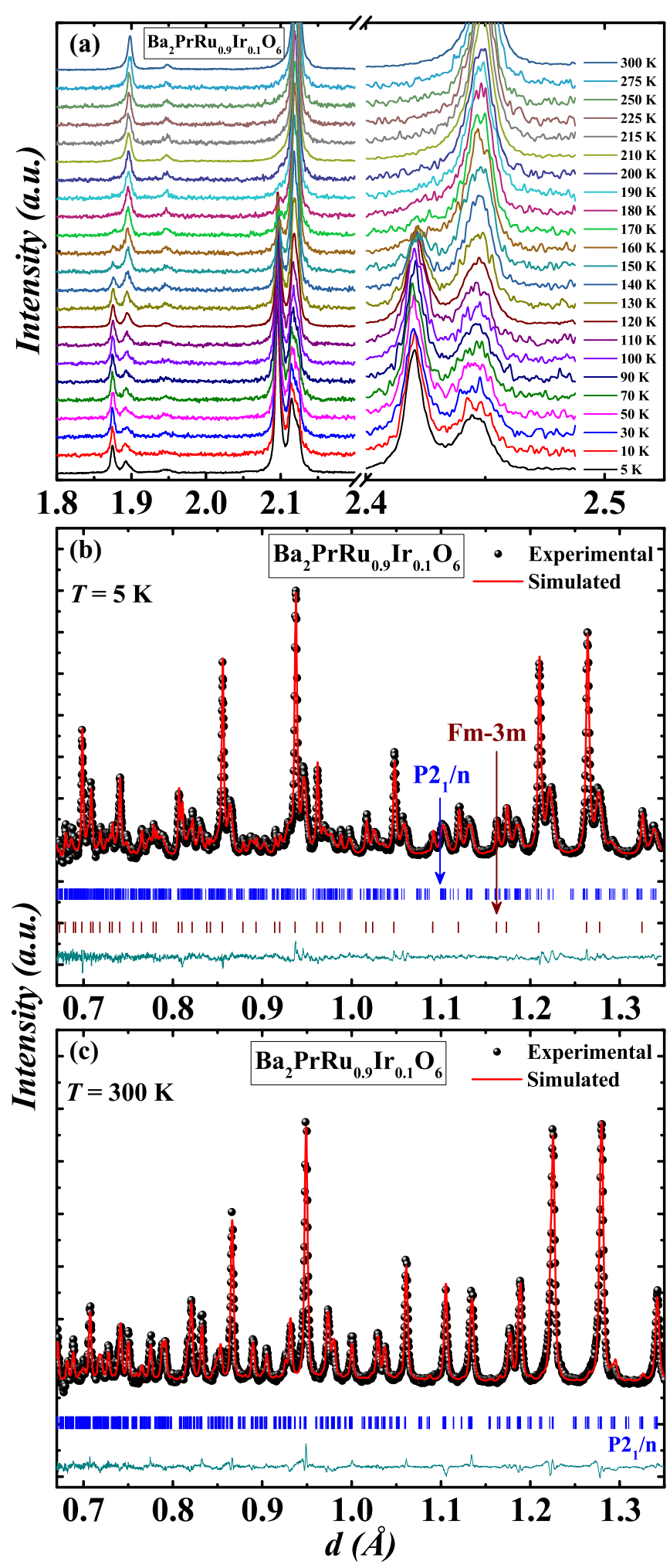

FIG. 2. (a) Powder neutron diffraction patterns of $\mathrm{Ba}_{2} \mathrm{PrRu}_{0.9} \mathrm{Ir}_{0.1} \mathrm{O}_{6}$ obtained from HRPD in the temperature range 5 to $300 \mathrm{~K}$. Panels (b) and (c) show Rietveld refinements of diffraction data measured at $5 \mathrm{~K}$ and $300 \mathrm{~K}$, respectively. Black circles indicate the experimental data points and red solid lines are the simulated curves. The Bragg peak positions of the monoclinic and cubic phases are indicated by blue and maroon vertical ticks, respectively, and the light-blue line shows the difference between the data and the simulated curve. 

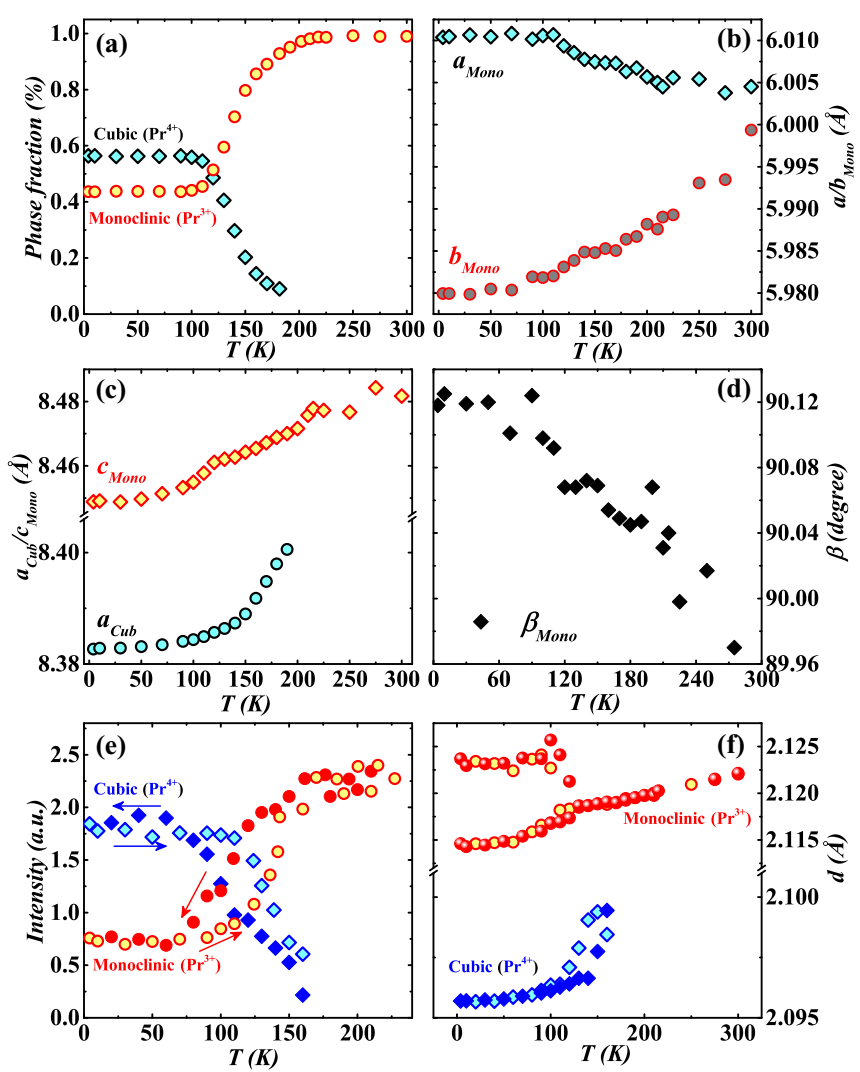

FIG. 3. Temperature dependence of structural parameters of $\mathrm{Ba}_{2} \operatorname{PrRu}_{0.9} \mathrm{Ir}_{0.1} \mathrm{O}_{6}$. (a) Fraction of monoclinic (space group $P 2_{1} / n$ ) and cubic (space group $F m \overline{3} m$ ) phases. (b) Monoclinic lattice parameters $a_{\text {mono }}$ and $b_{\text {mono }}$. (c) Monoclinic lattice parameter $c_{\text {mono }}$ and cubic lattice parameter $a_{\text {cub }}$. (d) Temperature variation of the monoclinic angle $\beta_{\text {mono }}$. (e) Single peak analysis which shows the variation of the fraction of monoclinic phase (containing $\mathrm{Pr}^{3+}$ ) and cubic phase (containing $\mathrm{Pr}^{4+}$ ) in warming and cooling measurements. (f) The $d$ spacing of the peaks shown in (e).

lattice parameters of the monoclinic phase start to change at about $100 \mathrm{~K}$, such that at $300 \mathrm{~K} a_{\text {mono }} \approx b_{\text {mono }} \approx c_{\text {mono }} / \sqrt{2}$ and $\beta_{\text {mono }} \approx 90^{\circ}$. This implies that the structure is nearly cubic at $300 \mathrm{~K}$.

The temperature dependence of the coexistence of the monoclinic and the cubic phases was investigated further by fitting the intensity of individual representative Bragg peaks from both phases during both heating and cooling cycles; see Figs. 3(e) and 3(f). This analysis clearly reveals the presence of a thermal hysteresis between $80 \mathrm{~K}$ and $170 \mathrm{~K}$, which confirms the first-order nature of this transition.

\section{Magnetic structure}

The magnetic susceptibility and structural studies clearly reveal the first-order valence phase transition in $\mathrm{Ba}_{2} \operatorname{PrRu}_{0.9} \mathrm{Ir}_{0.1} \mathrm{O}_{6}$ below approximately $200 \mathrm{~K}$. In Fig. 4 we present neutron diffraction results on the magnetic structure of $\mathrm{Ba}_{2} \mathrm{PrRu}_{0.9} \mathrm{Ir}_{0.1} \mathrm{O}_{6}$. The appearance of Bragg peaks at positions where no intensity from the structure of the two phases is present can be related to the long range order of magnetic moments. A rapid scan taken on cooling locates the onset of magnetic order at $T_{\mathrm{N}} \simeq 110 \mathrm{~K}$. The positions of
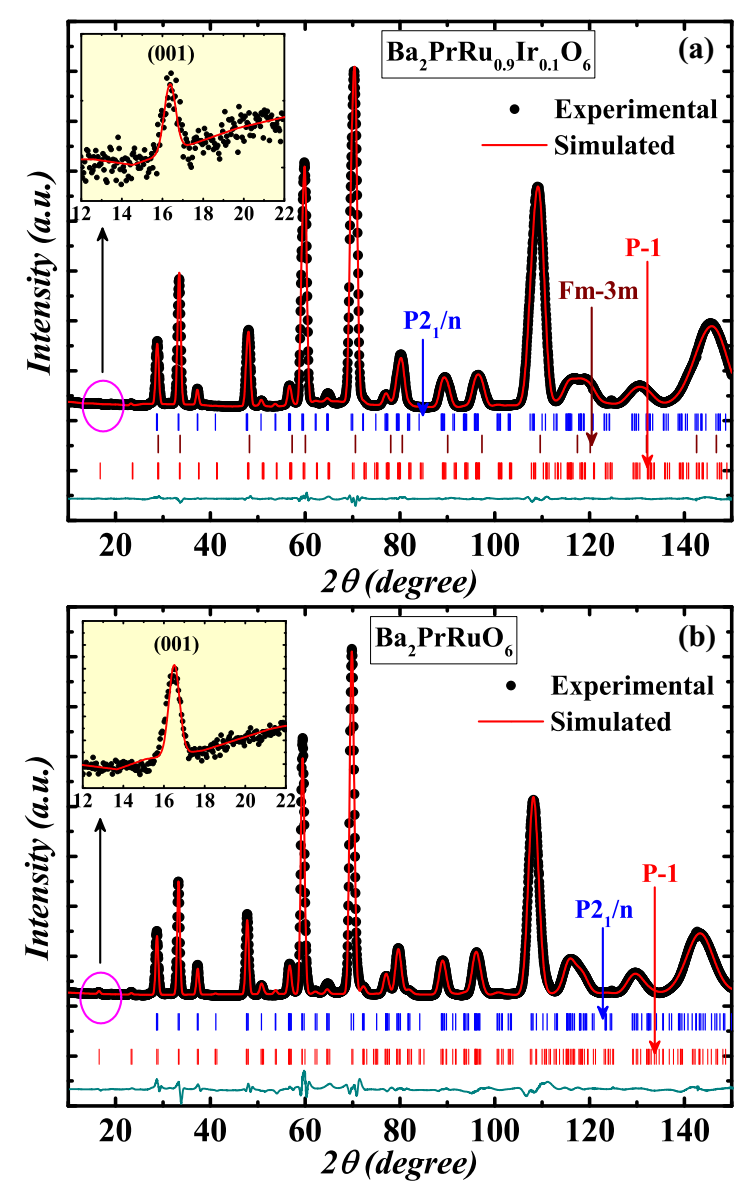

FIG. 4. (a) Rietveld refinement of powder neutron diffraction data recorded on D20 at $2 \mathrm{~K}$. Black points indicate the experimental data points and the red solid line is the simulated curve. The structural Bragg reflections are indicated by blue and maroon vertical ticks for the monoclinic and cubic phases, respectively, and red ticks mark the magnetic Bragg reflections from the monoclinic phase. Inset: the (001) magnetic reflection together with fitted peak. Panel (b) shows the same for $\mathrm{Ba}_{2} \mathrm{PrRuO}_{6}$ with blue and red ticks for the structural (monoclinic) and magnetic Bragg reflections.

the magnetic peaks are compatible with a propagation vector $\mathbf{q}=(0,0,0)$ of the monoclinic phase. Magnetic symmetry analysis was performed with the Basireps software [25,26] to determine the allowed irreducible representations (irreps) for the Pr sublattice occupying the Wyckoff position $2 c$ and the $\mathrm{Ru}$ sublattice at $2 d$. There are only two allowed irreps, the same for both sites, which link the symmetry-related atomic positions antiferromagnetically in the $a$ and $c$ directions, and ferromagnetically in the $b$ direction, or vice versa. Only the first coupling scheme can account for the magnetic peak intensities, corresponding to the type-I antiferromagnetic structure (magnetic space-group $P 2_{1} / c$ ) found previously $[19,20]$ for $\mathrm{Ba}_{2} \mathrm{PrRuO}_{6}$. The FullProf software [27] was used to fit the $2 \mathrm{~K}$ diffraction data, and a stable refinement [see Fig. 4(a)] was achieved assuming the presence of two structural phases and one magnetic phase (the monoclinic structural phase, which orders magnetically, and the cubic structural phase for which no magnetic ordering was detected in our neutron diffraction study at $2 \mathrm{~K}$ ). This 


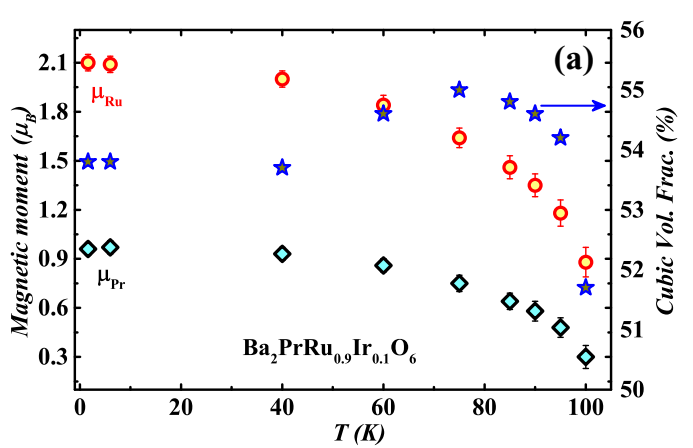

(b)

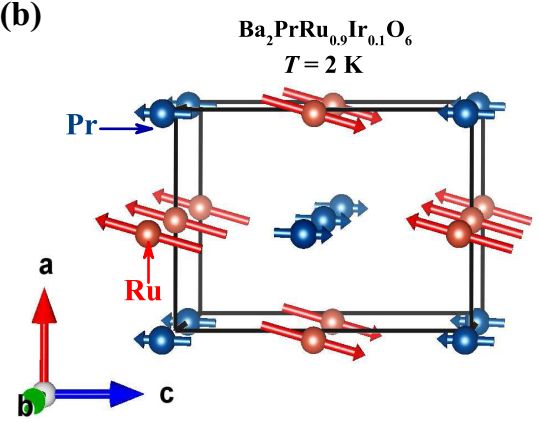

(c)

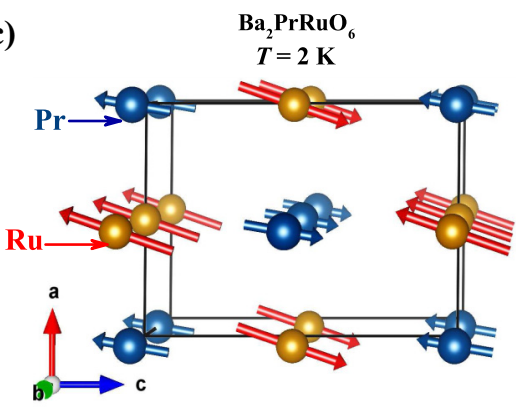

FIG. 5. (a) Temperature dependence of $\mathrm{Pr}$ and Ru moment of the monoclinic phase in $\mathrm{Ba}_{2} \operatorname{PrRu}_{0.9} \mathrm{Ir}_{0.1} \mathrm{O}_{6}$. Blue stars indicate the volume fraction of the cubic phase (paramagnetic) with temperature. Panels (b) and (c) depict the alignment of Pr and Ru spins in a magnetic unit cell of $\mathrm{Ba}_{2} \operatorname{PrRu}_{0.9} \mathrm{Ir}_{0.1} \mathrm{O}_{6}$ and $\mathrm{Ba}_{2} \mathrm{PrRuO}_{6}$, respectively.

has to be compared to the case of $\mathrm{Ba}_{2} \mathrm{PrRuO}_{6}$ for which a good refinement could be achieved considering only one monoclinic structural phase and one magnetic phase, as displayed in Fig. 4(b).

Both the Pr and Ru sites carry a magnetic moment which is preferentially aligned along the $c$ axis. Figure 5(a) shows the temperature dependence of the refined Pr and Ru moments and of the volume fraction of the cubic phase of $\mathrm{Ba}_{2} \mathrm{PrRu}_{0.9} \mathrm{Ir}_{0.1} \mathrm{O}_{6}$ as determined from the three-phase refinement of the D20 data. As the magnetic form factor of the $\mathrm{Ru}^{5+}$ ion is not listed in the International Tables of Crystallography an empirical magnetic form factor which had been determined previously [28] has been used. The plot shows that at about $100 \mathrm{~K}$, where the volume fraction of the cubic phase starts to decrease rapidly, the magnetic moments of Pr and Ru have decreased to nearly zero. As noted above, the magnetic Pr and $\mathrm{Ru}$ sublattices couple ferromagnetically in the $a b$ plane and these ferromagnetic layers are aligned antiferromagnetically along the $c$ axis as shown in Fig. 5(b).
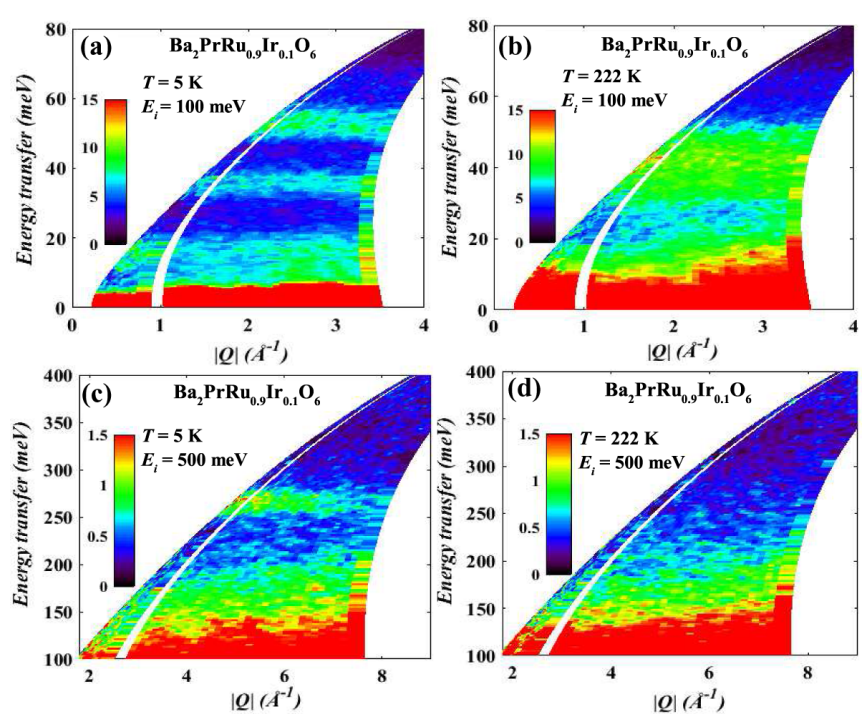

FIG. 6. Neutron scattering spectra of $\mathrm{Ba}_{2} \operatorname{PrRu}_{0.9} \mathrm{Ir}_{0.1} \mathrm{O}_{6}$. Panels (a) and (b) show color-coded intensity maps as a function of energy transfer $(\hbar \omega)$ and momentum transfer $(|\mathbf{Q}|)$ at $5 \mathrm{~K}$ and $222 \mathrm{~K}$, respectively, measured with an incident energy $E_{\mathrm{i}}=100 \mathrm{meV}$. Panels (c) and (d) display the same plots for $E_{\mathrm{i}}=500 \mathrm{meV}$.

The existence of a magnetic component along the $a$ direction can be inferred from the presence of the purely magnetic (001) peak; see the insets of Figs. 4(a) and 4(b). Because of the weakness of this peak, a refinement of the purely magnetic diffraction was undertaken for $\mathrm{Ba}_{2} \mathrm{PrRu}_{0.9} \mathrm{Ir}_{0.1} \mathrm{O}_{6}$ using a difference data set $2-130 \mathrm{~K}$, where the scale factor was fixed to the value obtained for the monoclinic phase fraction at $2 \mathrm{~K}$. A refinement which allowed the presence of a magnetic component in $a$ direction on both sublattices did not converge as the Pr component fluctuates around zero. We therefore fixed the $a$ component for Pr to zero leading to a stable refinement with $\mu_{\mathrm{Pr}}^{c}=1.10(1) \mu_{\mathrm{B}}, \mu_{\mathrm{Ru}}^{a}=0.55(3) \mu_{\mathrm{B}}$, and $\mu_{\mathrm{Ru}}^{c}=2.02(1) \mu_{\mathrm{B}}$. A similar refinement of a difference data set $2-150 \mathrm{~K}$ was performed for the parent compound $\mathrm{Ba}_{2} \mathrm{PrRuO}_{6}$. This time, the presence of a magnetic component in the $a$ direction could be ascertained for $\mathrm{Pr}$ as well as for $\mathrm{Ru}$ : $\mu_{\mathrm{Pr}}^{a}=0.26(9) \mu_{\mathrm{B}}, \mu_{\mathrm{Pr}}^{c}=1.09(1) \mu_{\mathrm{B}}, \mu_{\mathrm{Ru}}^{a}=0.30(9) \mu_{\mathrm{B}}$, and $\mu_{\mathrm{Ru}}^{c}=1.88(1) \mu_{\mathrm{B}}$ [Fig. 5(c)]. We note that, for $\mathrm{Ba}_{2} \mathrm{PrRuO}_{6}$, previous reports mention only a $c$-axis component for both sublattices $[19,20]$. Further, from the temperature dependence of the magnetic moments of the $\mathrm{Ru}$ and $\mathrm{Pr}^{3+}$ ions given in Fig. 5(a), it is clear that the magnetic ordering is driven by a co-operative effect of both sublattices $\left(\mathrm{Ru}\right.$ and $\left.\mathrm{Pr}^{3+}\right)$ and not by the magnetic ordering in one sublattice that drives the magnetic ordering in the other sublattice. Furthermore, RKKY exchanges are generally observed in metals employing an interaction through the conduction electrons. In the present oxide compounds, the layers made of $\mathrm{PrO}_{6}$ and $(\mathrm{Ru} / \mathrm{Ir}) \mathrm{O}_{6}$ octahedra are separated by $\mathrm{Ba}$. Hence superexchange interaction via oxygen is favorable here.

\section{Inelastic neutron scattering study}

Figure 6 presents color-coded INS intensity maps as a function of energy transfer $(\hbar \omega)$ and momentum $\operatorname{transfer}(Q=$ 

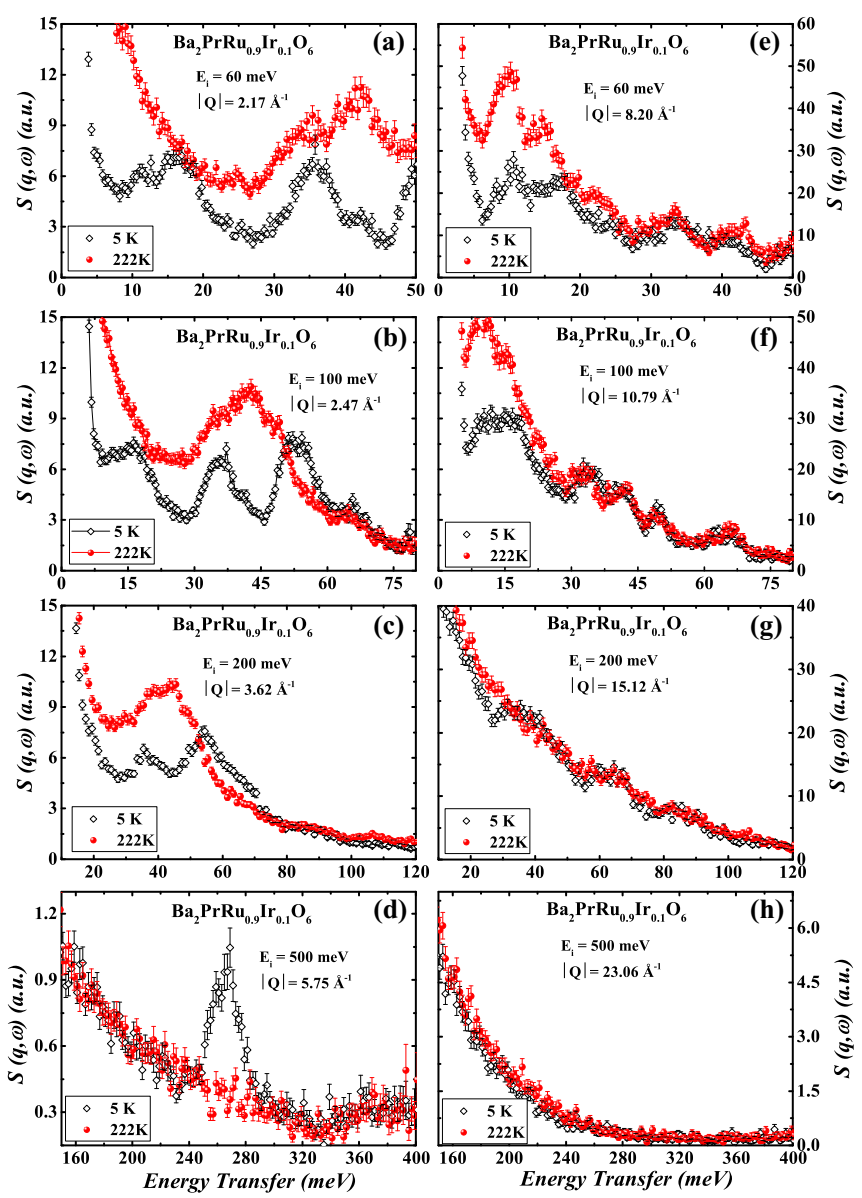

FIG. 7. $Q$-averaged INS intensity as a function of energy transfer at different $E_{i}(=60,100,200$, and $500 \mathrm{meV})$. Left and right panels show the low- $Q$ and high- $Q$ regimes, respectively.

$|\mathbf{Q}|)$ for $E_{\mathrm{i}}=100 \mathrm{meV}$ and $500 \mathrm{meV}$ at $T=5$ and $222 \mathrm{~K}$. The $E_{\mathrm{i}}=100 \mathrm{meV}$ spectrum at $5 \mathrm{~K}$, Fig. 6(a), shows four dispersionless bands of scattering at $\hbar \omega=15,35,55$, and $68 \mathrm{meV}$, while the $E_{\mathrm{i}}=500 \mathrm{meV}$ spectrum at $5 \mathrm{~K}$, Fig. 6(c), contains a dispersionless band at $\hbar \omega=264 \mathrm{meV}$. The $264 \mathrm{meV}$ excitation is absent at $222 \mathrm{~K}$, Fig. 6(d), while the excitations between 35 and $68 \mathrm{meV}$ transform from four well-defined peaks into a very broad (in energy) signal centered near $45 \mathrm{meV}$ at $222 \mathrm{~K}$, Fig. 6(b). The lack of dispersion with $Q$, and the reduction in intensity with $Q$ consistent with a magnetic form factor, implies that these signals arise from localized crystalline electric field (CEF) transitions of the Pr ions.

Figures $7(\mathrm{a})-7(\mathrm{~d})$ show spectra measured at different $E_{\mathrm{i}}$ from 60 to $500 \mathrm{meV}$, averaged over a small range of $Q$ in the low $Q$ regime. Data for both $T=5 \mathrm{~K}$ and $222 \mathrm{~K}$ are included. These spectra confirm that at $222 \mathrm{~K}$ (also at $130 \mathrm{~K}$ not shown here) there is a single broad peak centered near $45 \mathrm{meV}$, whereas at $5 \mathrm{~K}$ four well-defined peaks appear at around $15,35,55$, and $68 \mathrm{meV}$ (also observed at $65 \mathrm{~K}$, not shown here). In the $500 \mathrm{meV}$ spectra, Fig. 7(d), the peak at $\hbar \omega=264 \mathrm{meV}$ in the $5 \mathrm{~K}$ spectrum is completely absent from the $222 \mathrm{~K}$ spectrum.

We identify the $264 \mathrm{meV}$ peak as a CEF transition within the $J=5 / 2$ ground state level of $\operatorname{Pr}^{4+}$ in the cubic phase, and the four low-energy peaks as CEF transitions of $\mathrm{Pr}^{3+}$ in
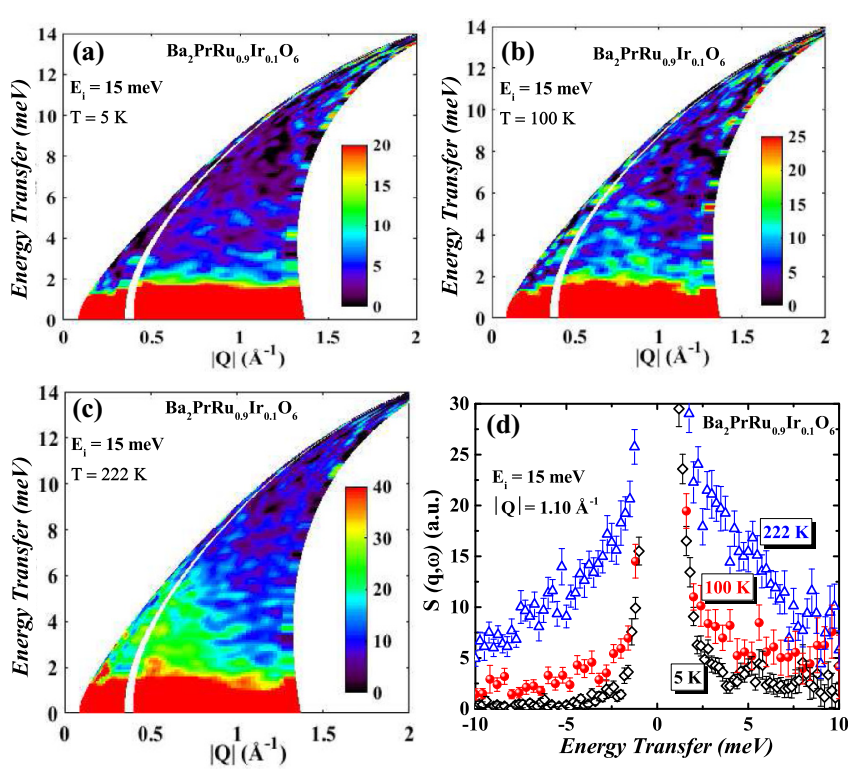

FIG. 8. INS spectrum of $\mathrm{Ba}_{2} \operatorname{PrRu}_{0.9} \mathrm{Ir}_{0.1} \mathrm{O}_{6}$ measured on HET with $E_{\mathrm{i}}=15 \mathrm{meV}$. Panels (a), (b), and (c) show the color-coded intensity maps as a function of energy transfer and $Q$ recorded at $5 \mathrm{~K}, 100 \mathrm{~K}$, and $222 \mathrm{~K}$. Panel (d) is the $Q$-averaged scattering at the same temperatures as a function of energy transfer.

the monoclinic phase. This interpretation follows from the observations described earlier, that the monoclinic phase is present both above and below the valence transition temperature $T_{\mathrm{V}} \simeq 170 \mathrm{~K}$, whereas the cubic phase is only present at $T<T_{\mathrm{V}}$.

Further evidence to support the assignment of the $264 \mathrm{meV}$ peak comes from a comparison with the INS spectrum of $\mathrm{BaPrO}_{3}$, which contains a peak at $255 \mathrm{meV}$ [29-31]. In $\mathrm{BaPrO}_{3}$, the $\mathrm{Pr}^{4+}$ ions have a slightly distorted octahedral coordination very similar to that in the monoclinic phase of $\mathrm{Ba}_{2} \mathrm{PrRu}_{0.9} \mathrm{Ir}_{0.1} \mathrm{O}_{6}$. The $255 \mathrm{meV}$ in $\mathrm{BaPrO}_{3}$ was found to originate from the $\Gamma_{7}$ to $\Gamma_{8}$ transitions within the $J=5 / 2$ level [29]. Furthermore, we have observed a similar CEF excitation at $276 \mathrm{meV}$ in $\mathrm{Ba}_{2} \mathrm{PrIrO}_{6}$ [24], also attributed to the same group of $\Gamma_{7}$ to $\Gamma_{8}$ transitions of $\mathrm{Pr}^{4+}$.

Figures 7(e)-7(h) show spectra for averaged $Q$ values in the high $Q$ regime. In this regime the spectra are dominated by phonon and mutiphonon scattering. As expected, the low energy part of the spectra increase in intensity with temperature due to thermal population of phonons. There are also some small changes in the peak positions and widths between $5 \mathrm{~K}$ and $222 \mathrm{~K}$ which are possibly, but not conclusively, associated with the structural transition.

Figure 8 shows INS spectra measured with $E_{\mathrm{i}}=15 \mathrm{meV}$. The spectrum at $T=100 \mathrm{~K}$ shows a small increase in scattering compared with that at $5 \mathrm{~K}$, but at $222 \mathrm{~K}$ the spectrum displays strong additional quasielastic scattering. As discussed earlier, $222 \mathrm{~K}$ is above both the magnetic ordering transition $\left(T_{\mathrm{N}} \simeq 110 \mathrm{~K}\right)$ and the valence transition $\left(T_{\mathrm{V}} \simeq\right.$ $170 \mathrm{~K}$ ), and at $222 \mathrm{~K}$ there exists only a single structural phase. We attribute the quasielastic signal observed at $222 \mathrm{~K}$, therefore, to paramagnetic scattering from the $\mathrm{Pr}^{3+}$ and $\mathrm{Ru}^{5+}$ ions in the monoclinic phase. The reduction in scattering on 
cooling, first to $100 \mathrm{~K}$ and then $5 \mathrm{~K}$, could be accounted for by the freezing of dynamic magnetic fluctuations due to magnetic order and a reduction in the volume fraction of the monoclinic phase below $T_{\mathrm{V}}$. The significant widths of both the quasielastic scattering and the inelastic peaks observed at $222 \mathrm{~K}$ may be due to residual magnetic correlations above $T_{\mathrm{N}}$ and/or to lifetime damping due to magnetoelastic effects or valence fluctuations associated with the incipient valence transition. We have also measured pure $\mathrm{Ba}_{2} \mathrm{PrRuO}_{6}$ with the same $E_{\mathrm{i}}$ values and we find very similar changes in the excitations when going from $147 \mathrm{~K}$ to $7 \mathrm{~K}$ (data not shown here).

We now present a more quantitative analysis of the CEF splitting of the Pr ions in order to provide further support for our interpretation of the spectra. We consider first the $\mathrm{Pr}^{4+}$ ions in the cubic phase. The $4 f^{1}$ configuration of $\mathrm{Pr}^{4+}$ is split by the spin-orbit interaction $(\zeta=107.2 \mathrm{meV})$ into two levels, ${ }^{2} F_{5 / 2}(J=5 / 2)$ and ${ }^{2} F_{7 / 2}(J=7 / 2)$ with sixfold and eightfold degeneracy $(=2 J+1)$, respectively. The degeneracy is lifted by the $\mathrm{CEF}$ from the neighboring ions, which has octahedral point symmetry $\left(m \overline{3} m\right.$ or $\left.O_{h}\right)$ and is described by the Hamiltonian

$$
\mathcal{H}_{\mathrm{CEF}}=B_{0}^{4}\left[C_{0}^{(4)} \pm \frac{\sqrt{ } 70}{14} C_{4}^{(4)}\right]+B_{0}^{6}\left[C_{0}^{(6)} \mp \frac{\sqrt{ } 14}{2} C_{4}^{(6)}\right],
$$

where $B_{q}^{k}$ are phenomenological CEF parameters and $C_{q}^{(k)}(\theta, \phi)=\sqrt{\frac{4 \pi}{(2 k+1)}} Y_{k, q}(\theta, \phi)$ are tensor operators as defined in Ref. [32], with $Y_{k, q}(\theta, \phi)$ the spherical harmonics. The relation between the CEF parameters of the tensor operators (i.e., the $B_{q}^{k}$ used here) and the commonly used Stevens operator equivalents can be found in Ref. [33], although we caution that the Stevens operator method is not accurate for the present system because the CEF splitting is comparable to the spin-orbit splitting.

The cubic CEF described by (1) splits the ground state ${ }^{2} F_{5 / 2}$ level of $\mathrm{Pr}^{4+}$ into a doublet of $\Gamma_{7}$ symmetry and a quartet of $\Gamma_{8}$ symmetry, and splits the ${ }^{2} F_{7 / 2}$ upper level into two doublets $\left(\Gamma_{6}^{\prime}\right.$ and $\left.\Gamma_{7}^{\prime}\right)$ and a quartet $\left(\Gamma_{8}^{\prime}\right)$. The prime notation is used here to distinguish the states belonging to ${ }^{2} F_{7 / 2}$ from those of ${ }^{2} F_{5 / 2}$.

In Fig. 9 we show the INS spectra recorded with the two highest $E_{\mathrm{i}}$ values. The experimental spectra are magnified to emphasize the weak features, and are plotted for a fixed average scattering angle $\phi$ rather than for a fixed average $Q$. The lines are simulated spectra calculated from Eq. (1) with the program SPECTRE [34] and the cross-section formulas summarized in Ref. [35]. The ratio $B_{0}^{6} / B_{0}^{4}$ was initially fixed by the point charge model for nearest neighbors only, and $B_{0}^{4}$ was varied to put the $\Gamma_{8}$ level at $264 \mathrm{meV}$. As well as the $\Gamma_{7}$ to $\Gamma_{8}$ transition at $264 \mathrm{meV}$, the calculation predicted an interlevel transition from $\Gamma_{7}$ to $\Gamma_{7}^{\prime}$ at around $420 \mathrm{meV}$. This coincides with a small peak in the data centered on about 400 meV; see Fig. 9(a). A similar feature was observed in the INS spectrum of $\mathrm{Ba}_{2} \mathrm{PrIrO}_{6}$ at about $370 \mathrm{meV}$ [24]. Subsequently, $B_{0}^{6}$ was allowed to vary slightly. The simulations shown in Figs. 9(a) and 9(b) are calculated with CEF parameters (in Wybourne tensor notation) $B_{0}^{4}=971 \mathrm{meV}$ and $B_{0}^{6}=60 \mathrm{meV}$, and Fig. 9(c) displays the full CEF level scheme of $\mathrm{Pr}^{4+}$ for these parameters.
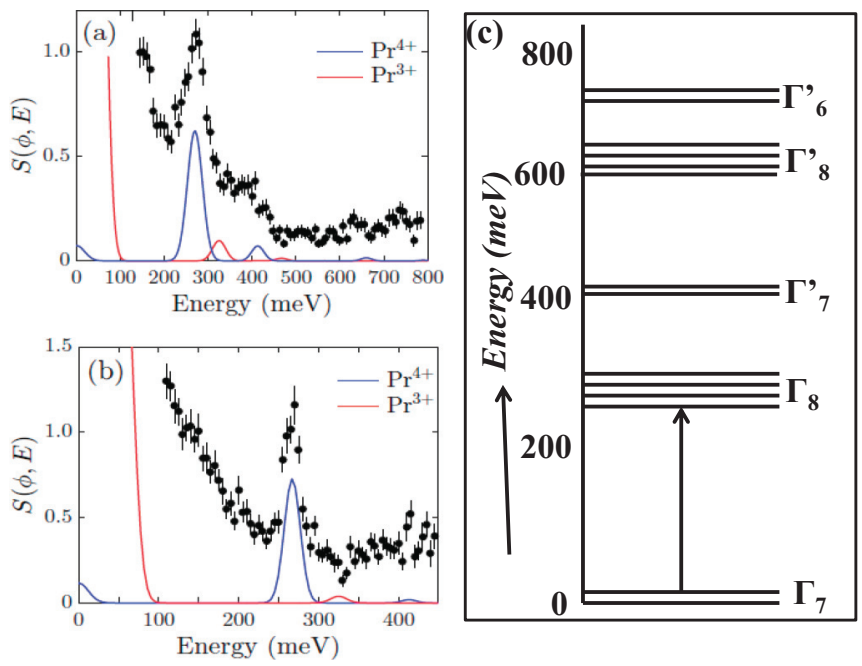

FIG. 9. INS spectra of $\mathrm{Ba}_{2} \mathrm{PrRu}_{0.9} \mathrm{Ir}_{0.1} \mathrm{O}_{6}$ at fixed scattering angle $\phi$ as a function of energy transfer at $5 \mathrm{~K}$, for incident energies of (a) $E_{\mathrm{i}}=900 \mathrm{meV}$, averaged from $\phi=3$ to $10^{\circ}\left(\langle\phi\rangle=6.5^{\circ}\right)$, and (b) $E_{\mathrm{i}}=500 \mathrm{meV}$, averaged from $\phi=3$ to $12^{\circ}\left(\langle\phi\rangle=7.5^{\circ}\right)$. The solid lines show the simulated INS spectra for $\operatorname{Pr}^{4+}$ (blue) and $\operatorname{Pr}^{3+}$ (red) calculated from the cubic CEF model described in the text. (c) Energy level scheme for the $J=5 / 2$ and $J=7 / 2$ levels of $\operatorname{Pr}^{4+}$ calculated from the CEF model.

The point symmetry for the $\operatorname{Pr}^{3+}$ ions $\left(4 f^{2}\right)$ in the monoclinic phase is triclinic $\left(\overline{1}\right.$ or $\left.C_{i}\right)$. In such a low symmetry the CEF Hamiltonian required 15 parameters, and the ground state ${ }^{3} \mathrm{H}_{4}$ level is maximally split into nine singlets. Given that we have observed only four CEF transitions at $T=5 \mathrm{~K}$, a quantitative analysis of the $\mathrm{Pr}^{3+}$ data is not feasible. To gain a rough estimate of the contribution from $\mathrm{Pr}^{3+}$ to the high energy spectra we estimated the $\mathrm{Pr}^{3+}$ CEF parameters by scaling those for $\mathrm{Pr}^{4+}$ using relations applicable to the point charge model. A simulation of the $\mathrm{Pr}^{3+}$ spectrum obtained this way is shown on Figs. 9(a) and 9(b). We assumed $\mathrm{Pr}^{4+}$ and $\mathrm{Pr}^{3+}$ to be present in the ratio 0.58:0.42, as observed experimentally. The largest peak from $\mathrm{Pr}^{3+}$ in the high energy range is found at $330 \mathrm{meV}$, and corresponds to an interlevel transition from ${ }^{3} H_{4}$ to ${ }^{3} H_{5}$. This peak is nearly an order of magnitude smaller than the $264 \mathrm{meV}$ peak, and could contribute to the small peak observed in the data at around $400 \mathrm{meV}$. More importantly, the simulation confirms that the $264 \mathrm{meV}$ peak is from $\mathrm{Pr}^{4+}$, because there is no peak of comparable strength from $\mathrm{Pr}^{3+}$ in this energy range.

Finally, we remark that in $\mathrm{PrO}_{2}$, which also has $\mathrm{Pr}^{4+}$ in a cubic CEF environment, but in eightfold rather than sixfold coordination, the CEF-split levels are reversed, with $\Gamma_{8}$ as the ground state and $\Gamma_{7}$ as the excited state. In addition to the $\Gamma_{8}$ to $\Gamma_{7}$ transition near $130 \mathrm{meV}$ and interlevel transitions at higher energies, the INS spectrum of $\mathrm{PrO}_{2}$ contains broad magnetic peaks near $30 \mathrm{meV}$ and $160 \mathrm{meV}$ [36]. These broad continua are attributed to vibronic modes due to the dynamic Jahn-Teller effect. Such excitations occur in $\mathrm{PrO}_{2}$ because of the large orbital degeneracy of the $\Gamma_{8}$ ground state, and so are not expected to be observed for $\mathrm{Pr}^{4+}$ in octahedral coordination, as in $\mathrm{Ba}_{2} \mathrm{PrRu}_{0.9} \mathrm{Ir}_{0.1} \mathrm{O}_{6}$. 

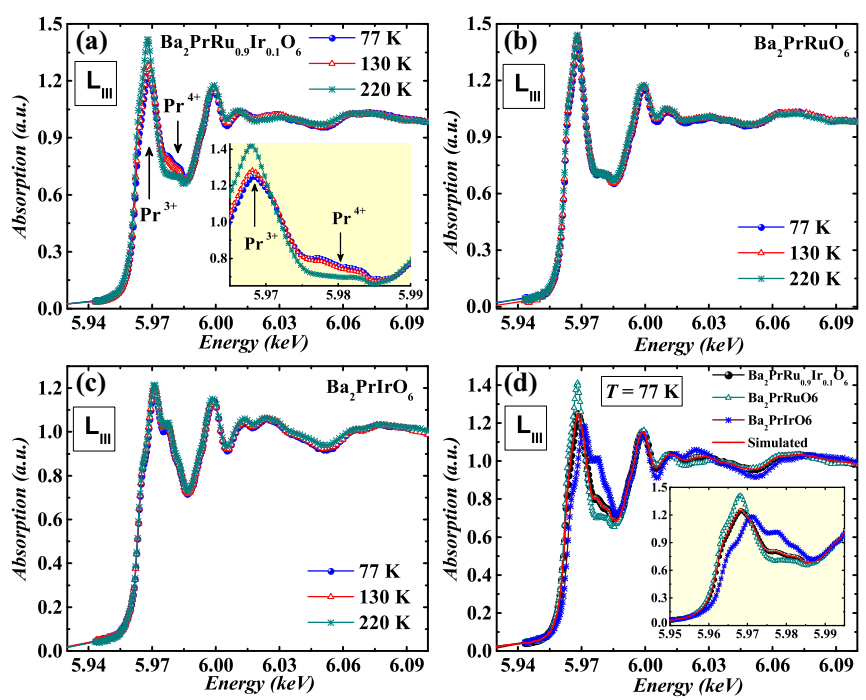

FIG. 10. XANES spectra at various temperatures are depicted for (a) $\mathrm{Ba}_{2} \mathrm{PrRu}_{0.9} \mathrm{Ir}_{0.1} \mathrm{O}_{6}$, (b) $\mathrm{Ba}_{2} \mathrm{PrRuO}_{6}$, and (c) $\mathrm{Ba}_{2} \mathrm{PrIrO}_{6}$. The inset of (a) shows an enlarged view of the anomalies near $5.966 \mathrm{keV}$ and $5.979 \mathrm{keV}$. Panel (d) shows the calculated spectra of $\mathrm{Ba}_{2} \mathrm{PrRu}_{0.9} \mathrm{Ir}_{0.1} \mathrm{O}_{6}$ from the observed spectra of $\mathrm{Ba}_{2} \mathrm{PrRuO}_{6}$ and $\mathrm{Ba}_{2} \mathrm{PrIrO}_{6}$ at $77 \mathrm{~K}$. The inset of (d) shows an enlarged view of the anomalies near $5.95 \mathrm{keV}$ and $5.99 \mathrm{keV}$.

\section{X-ray absorption spectroscopy}

We have investigated the $\operatorname{Pr} L_{3}$-edge $\mathrm{x}$-ray absorption nearedge structure (XANES) spectrum of $\mathrm{Ba}_{2} \mathrm{PrRu}_{1-x} \mathrm{Ir}_{x} \mathrm{O}_{6}$ with $x=0.1$ in order to obtain direct information on the valence states of the $\mathrm{Pr}$ ion below and above the transition. As a reference, we have also measured the compounds with $x=0$ and $x=1$. The $\operatorname{Pr} L_{3}$-edge measurements were carried out as a function of temperature, above and below the first-order phase transition. Figures 10 (a) to 10 (c) show $\operatorname{Pr}-L_{3}$ measurements at $77 \mathrm{~K}, 130 \mathrm{~K}$, and $220 \mathrm{~K}$ (data were collected during a heating cycle) for $\mathrm{Ba}_{2} \operatorname{PrRu}_{1-x} \operatorname{Ir}_{x} \mathrm{O}_{6}$ with $x=0.1,0$, and 1 , respectively.

It can be seen in Figs. 10(b) and 10(c) that the spectra of $\mathrm{Ba}_{2} \mathrm{Pr}^{3+} \mathrm{RuO}_{6}$ and $\mathrm{Ba}_{2} \mathrm{Pr}^{4+} \mathrm{IrO}_{6}$ do not show any temperature dependence, whereas those of $\mathrm{Ba}_{2} \mathrm{PrRu}_{0.9} \mathrm{Ir}_{0.1} \mathrm{O}_{6}$ exhibit a strong temperature dependence near $5.966 \mathrm{keV}$ $\left(L_{3}\right.$ of $\left.\mathrm{Pr}^{3+}\right)$ and $5.979 \mathrm{keV}\left(L_{3}\right.$ of $\left.\mathrm{Pr}^{4+}\right)$. The peak at $5.966 \mathrm{keV}$ arises due to the excitation from $2 p_{3 / 2}$ to $4 f^{2} 5 d^{*}$, which represents $\operatorname{Pr}^{3+}$ ions. The other feature near $5.979 \mathrm{keV}$ emerges from $2 p_{3 / 2}$ to $4 f^{2} \underline{L} 5 d^{*}$ ( $\underline{L}$ being the ligand hole) and $4 f^{1} 5 d^{*}$ excitations, which represent $\operatorname{Pr}^{4+}$ ions [37,38]. The intensity near $5.979 \mathrm{keV}$ is higher at $77 \mathrm{~K}$ and $130 \mathrm{~K}$ than at $220 \mathrm{~K}$, which indicates directly that at low temperatures the Pr valence changes from $3+$ to $4+$. This is also evidenced from the decrease in the intensity of the $5.966 \mathrm{eV}$ peak at $130 \mathrm{~K}$ and $77 \mathrm{~K}$. The inset of Fig. 10(a) shows clearly this temperature dependence. A very similar behavior was also observed for the $\operatorname{Pr} L_{2}$ edges (data not shown here).

To estimate the volume fraction of the $\operatorname{Pr}^{3+}$ and $\operatorname{Pr}^{4+}$ phases at $77 \mathrm{~K}$ in $\mathrm{Ba}_{2} \operatorname{PrRu}_{0.9} \mathrm{Ir}_{0.1} \mathrm{O}_{6}$, we have added the intensity of the two end members (i.e., $x=0$ and 1), $y \mathrm{Ru}+(1-y) \mathrm{Ir}$, and compared the calculated intensity with the observed intensity of the $x=0.1$ composition. The result is shown in Fig. 10(d). The observed and calculated intensities agree well for $y \approx 0.44$. This indicates that the volume fraction of the $\operatorname{Pr}^{4+}$ phase, i.e., $(1-y)$, is approximately $56 \%$ and that of $\mathrm{Pr}^{3+}$ phase is approximately $44 \%$ at $77 \mathrm{~K}$, which is in agreement with that estimated from the neutron diffraction data discussed above.

\section{CONCLUSIONS}

We have investigated $\mathrm{Ba}_{2} \operatorname{PrRu}_{1-x} \mathrm{Ir}_{x} \mathrm{O}_{6}(x=0.1)$ with neutron diffraction, inelastic neutron scattering, and $x$-ray absorption measurements, as well as susceptibility and heat capacity, and compared the results with $x=0$ and 1. All our results confirm the first-order nature of a valence phase transition of Pr below $200 \mathrm{~K}$ in $\mathrm{Ba}_{2} \operatorname{PrRu}_{1-x} \mathrm{Ir}_{x} \mathrm{O}_{6}(x=0.1)$.

Our high resolution neutron diffraction study at $300 \mathrm{~K}$ reveals that the $x=0.1$ sample is single phase and crystallized in the monoclinic phase, whereas below $170 \mathrm{~K}$ two phases coexist: the monoclinic phase (containing $\operatorname{Pr}^{3+}$ ) and a cubic phase (containing $\mathrm{Pr}^{4+}$ ). We have estimated the volume faction of these two phases and found $58 \%$ for the cubic and $42 \%$ for the monoclinic phase at $5 \mathrm{~K}$.

Furthermore, our neutron diffraction study clearly shows a long range magnetic ordering of both $\mathrm{Pr}$ and $\mathrm{Ru}$ moments below $100 \mathrm{~K}$ in the monoclinic phase. Analysis of the $x=0.1$ data at $2 \mathrm{~K}$ revealed that the Pr moments point along the $c$ axis, but the $\mathrm{Ru}$ moments have a minority $a$-axis component in addition to a majority $c$-axis component. On the other hand, for $x=0$ we have found that both $\mathrm{Pr}$ and $\mathrm{Ru}$ moments are tilted away from the $c$-axis.

Our high energy inelastic neutron scattering study on $x=$ 0.1 reveals an excitation at $264 \mathrm{meV}$ which is present at $5 \mathrm{~K}$ but absent at $222 \mathrm{~K}$, consistent with a change of the valence state of some of the $\mathrm{Pr}$ ions from $\mathrm{Pr}^{4+}$ at low temperature to $\mathrm{Pr}^{3+}$ above $200 \mathrm{~K}$, corresponding to a transition from cubic to monoclinic structures.

Finally, we have observed clear evidence for the Pr valence transition in our x-ray absorption measurements on $x=0.1$, in contrast to $x=0$ and 1 for which no valence transition was observed.

\section{ACKNOWLEDGMENTS}

We thank B. D. Rainford for interesting discussion, D. Visser for participating in the X-ray experiment, and Y. Izumiyama for help in the samples preparation. D.T.A. and A.D.H. would like to thank CMPC-STFC, Grant No. CMPC09108, for financial support. D.T.A. would like to thank JSPS for the invitation fellowship. J.S. would like to thank the European Union's Horizon 2020 research and innovation programme under the Marie Skodowska-Curie Grant Agreement (GA) No. 665593 awarded to the Science and Technology Facilities Council. A.T.B. is grateful for financial support from the U.K. Engineering and Physical Sciences Research Council, Grant No. EP/N034872/1. The authors would like to thank Diamond Light Source for beamtime on B18 (Proposal No. SP63810-1) and ISIS Facility for beam time on HRPD and HET (RB14882). 
[1] A. J. Freeman and R.-Q. Wu, J. Magn. Magn. Mater 100, 497 (1991).

[2] K. Yoshikawa, H. Sato, M. Arita, Y. Takeda, K. Hiraoka, K. Kojima, K. Tsuji, H. Namatame, and M. Taniguchi, Phys. Rev. B 72, 165106 (2005).

[3] T. Takabatake, F. Teshima, H. Fujii, S. Nishigori, T. Suzuki, T. Fujita, Y. Yamaguchi, J. Sakurai, and D. Jaccard, Phys. Rev. B 41, 9607 (1990).

[4] M. F. Hundley, P. C. Canfield, J. D. Thompson, Z. Fisk, and J. M. Lawrence, Phys. Rev. B 42, 6842 (1990).

[5] S. K. Malik and D. T. Adroja, Phys. Rev. B 43, 6277 (1991).

[6] R. Osborn, S. W. Lovesey, A. D. Taylor, and E. Black, in Handbook of Physics and Chemistry of Rare Earths, edited by K. A. Gschneidner, Jr. (Elsevier Science Publisher, B. V., Amsterdam, 1991), Vol. 14.

[7] J. M. Lawrence, P. S. Riseborough, and R. D. Parks, Rep. Prog. Phys. 44, 1 (1981).

[8] A. Jayaraman, V. Narayanamurti, E. Bucher, and R. G. Maines, Phys. Rev. Lett. 25, 1430 (1970).

[9] H. Wada, M. F. Hundley, R. Movshovich, and J. D. Thompson, Phys. Rev. B 59, 1141 (1999).

[10] W. Zhang, N. Sato, K. Yoshimura, A. Mitsuda, T. Goto, and K. Kosuge, Phys. Rev. B 66, 024112 (2002).

[11] I. Felner and I. Nowik, Phys. Rev. B 33, 617 (1986).

[12] I. Felner, I. Nowik, D. Vaknin, U. Potzel, J. Moser, G. M. Kalvius, G. Wortmann, G. Schmiester, G. Hilscher, E. Gratz, C. Schmitzer, N. Pillmayr, K. G. Prasad, H. de Waard, and H. Pinto, Phys. Rev. B 35, 6956 (1987).

[13] D. T. Adroja, B. D. Rainford, J. M. de Teresa, A. del Moral, M. R. Ibarra, and K. S. Knight, Phys. Rev. B 52, 12790 (1995).

[14] D. C. Koskenmaki and K. A. Gschneidner, Jr., in Handbook on the Physics and Chemistry of Rare Earths, edited by K. A. Gschneidner, Jr. and L. Eyring (North-Holland, Amsterdam, 1978), Chap. 4, p. 337.

[15] M. Wakeshima, Y. Izumiyama, Y. Doi, and Y. Hinatsu, Solid State Commun. 120, 273 (2001).

[16] D. T. Adroja and B. D. Rainford, Physica B 199, 498 (1994).

[17] H. Sato, K. Shimada, M. Arita, K. Hiraoka, K. Kojima, Y. Takeda, K. Yoshikawa, M. Sawada, M. Nakatake, H. Namatame, M. Taniguchi, Y. Takata, E. Ikenaga, S. Shin, K. Kobayashi, K. Tamasaku, Y. Nishino, D. Miwa, M. Yabashi, and T. Ishikawa, Phys. Rev. Lett. 93, 246404 (2004).

[18] L. Li and B. J. Kennedy, J. Solid State Chem. 177, 3290 (2004).

[19] Y. Izumiyama, Y. Doi, M. Wakeshima, Y. Hinatsu, Y. Shimojo, and Y. Morii, J. Phys.: Condens. Matter 13, 1303 (2001).
[20] N. G. Parkinson, P. D. Hatton, J. A. K. Howard, S. R. Giblin, I. Terry, C. Ritter, B.-H. Mok, and M.-K. Wu, J. Mater. Chem. 15, 1375 (2005).

[21] A. D. Hillier, D. T. Adroja, W. Kockelmann, M. Wakeshima, Y. Izumiyama, Y. Hinatsu, D. Visser, and B. D. Rainford, J. Magn. Magn. Mater. 310, e575 (2007).

[22] A. C. Larson and R. B. Von Dreele, General Structure Analysis System (GSAS), Los Alamos National Laboratory Report No. LAUR 86-748, 2000, https://permalink.lanl.gov/object/tr? what=info:lanl-repo/lareport/LA-UR-86-0748_REV.

[23] W. T. Fu and D. J. W. Ijdo, J. Solid State Chem. 178, 1312 (2007).

[24] W. Kockelmann, D. T. Adroja, A. D. Hillier, M. Wakeshima, Y. Izumiyama, Y. Hinatsu, K. S. Knight, D. Visser, and B. D. Rainford, Physica B 378-380, 543 (2006).

[25] J. Rodriguez-Carvajal, BASIREPS: a program for calculating irreducible representations of space groups and basis functions for axial and polar vector properties. Part of the FullProf suite of programs at www.ill.eu/sites/fullprof/.

[26] C. Ritter, Solid State Phenom. 170, 263 (2011).

[27] J. Rodriguez-Carvajal, Physica B 192, 55 (1993).

[28] N. Parkinson, P. D. Hatton, J. A. K. Howard, C. Ritter, F. Z. Chien, and M.-K. Wu, J. Mater. Chem. 13, 1468 (2003).

[29] S. Kern, C.-K. Loong, and G. H. Lander, Phys. Rev. B 32, 3051 (1985).

[30] D. J. Goosens, R. A. Robinson, and M. T. F. Telling, Physica B 352, 105 (2004).

[31] Y. Hinatsu, J. Solid State Chem. 119, 405 (1995).

[32] B. G. Wybourne, Spectroscopic Properties of Rare Earths (Interscience, New York, 1965).

[33] A. J. Kassman, J. Chem. Phys. 53, 4118 (1970).

[34] A. T. Boothroyd, SPECTRE, a program for calculating spectroscopic properties of rare earth ions in crystals (1990-2014), https://xray.physics.ox.ac.uk/software.htm.

[35] R. Osborn, S. W. Lovesey, and A. D. Taylor, in Handbook on the Physics and Chemistry of Rare Earths, edited by K. A. Gschneidner, Jr. and L. Eyring (Elsevier Science Publishing B. V., Amsterdam, 1991), Vol. 14, Chap. 93.

[36] A. T. Boothroyd, C. H. Gardiner, S. J. S. Lister, P. Santini, B. D. Rainford, L. D. Noailles, D. B. Currie, R. S. Eccleston, and R. I. Bewley, Phys. Rev. Lett. 86, 2082 (2001).

[37] H. Fujishiro, T. Naito, D. Takeda, N. Yoshida, T. Watanabe, K. Nitta, J. Hejtmánek, K. Knížek, and Z. Jirák, Phys. Rev. B 87, 155153 (2013).

[38] A. Bianconi, A. Marcelli, H. Dexpert, R. Karnatak, A. Kotani, T. Jo, and J. Petiau, Phys. Rev. B 35, 806 (1987). 